\title{
Valoración de los Programas de Mejora del Aprendizaje y del Rendimiento desde la visión de sus protagonistas en cuatro poblaciones de la provincia de Sevilla
}

\author{
Assessment of Learning Improvement and Performance Programs from the \\ viewpoint of its protagonists in four towns in the province of Seville
}

\author{
Maria del Carmen Corujo Vélez \\ mcorujo@us.es \\ Universidad de Sevilla. España \\ Sara Méndez García \\ sara.m.g@hotmail.es \\ Universidad de Sevilla. España
}

Ana María Rodríguez Carmona iD

anarodcar4@alum.us.es

Universidad de Sevilla. Ayuntamiento de El Viso del Alcor. España

\section{Resumen}

El trabajo es una aproximación descriptiva que pretende conocer los Programas de Mejora del Aprendizaje y del Rendimiento (PMAR), según la visión del alumnado, profesorado de los ámbitos y orientadores pertenecientes a 6 IES de la Provincia de Sevilla. Se utilizaron cuestionarios y entrevistas para la recogida de datos, que, posteriormente, fueron sometidos a un análisis de contenido y categorización. Los resultados obtenidos muestran que, en algunos casos, no se sigue la legislación de forma exhaustiva en la selección del alumnado y las valoraciones de los agentes implicados difieren notablemente respecto al programa, siendo las más favorables las de los estudiantes. En conclusión, son necesarias otras medidas para atender a todo el alumnado que no reúne algunos de los requisitos de acceso al PMAR.

Palabras clave: educación secundaria obligatoria; atención a la diversidad; agrupamientos flexibles; evaluación.

\section{Abstract}

The following work is a descriptive approach that seeks to know the Programs for the Improvement of Learning and Performance (PMAR), according to the vision of the students, teachers of the fields and counselors belonging to 6 IES of the Province of Seville. Questionnaires and interviews were used to collect data, which were subsequently subjected to content analysis and categorization. The results obtained show that, in some cases, the legislation is not followed exhaustively in the selection of students and the assessments of the agents involved differ markedly with respect to the program, being the most favorable those of the students. In conclusion, other measures are necessary to attend to all students who do not meet some of the requirements for access to PMAR.

Keywords: compulsory secondary education; attention to diversity; flexible groupings; evaluation.

Recibido / Received: 13-09-2017

Aceptado / Accepted: 05-03-2018

Cómo referenciar este artículo / How to reference this article:

Corujo, M.C., Méndez, S., \& Rodríguez, A.M. (2018). Valoración de los Programas de Mejora del Aprendizaje y del Rendimiento desde la visión de sus protagonistas en cuatro poblaciones de la provincia de Sevilla. Tendencias Pedagógicas, 32, 31-48. doi: 10.15366/tp2018.32.003 


\section{Introducción}

Las sucesivas leyes establecidas en nuestro país en las últimas décadas han tratado de responder a la diversidad de las aulas de diferentes formas, tanto en la etapa de Educación Infantil como en Primaria y Secundaria; centrándonos en esta última, donde la edad del alumnado, sus intereses y las dificultades encontradas se multiplican exponencialmente, trataremos de hacer un breve recorrido sobre las mismas, para después incidir en los Programas de Mejora para el Aprendizaje y el Rendimiento (PMAR, en lo sucesivo), que es el tema que nos ocupa.

La L.O.G.S.E. (Ley Orgánica de Ordenación General del Sistema Educativo) de 1990, además de las medidas ordinarias para atender a la diversidad en la Enseñanza Secundaria Obligatoria (E.S.O.), tales como un diseño curricular que contemple la diversidad, la introducción de nuevos contenidos, metodologías, criterios e instrumentos de evaluación, actividades diferenciadas, agrupamientos flexibles, entre otras, también propuso unas medidas específicas donde se incluía la optatividad y opcionalidad de diferentes asignaturas, la adaptación y la diversificación curricular. Para dar respuesta a esta última, se establecieron los Programas de Diversificación Curricular (P.D.C.), una medida excepcional que consiste en adaptar globalmente el currículo de la E.S.O. a las necesidades individuales de determinados alumnos y alumnas, con una organización distinta a la establecida con carácter general, que ha de atender a las capacidades generales de los objetivos de la etapa y, que se aplica si todas las medidas anteriores han fracasado, y se cumplen una serie de requisitos por parte del alumnado.

Centrándonos en el tema del agrupamiento del alumnado, este recurso supuso un cambio en este sentido, ya que el alumnado (inicialmente de $4^{\circ}$ y posteriormente desde $3^{\circ}$ ) pertenecía simultáneamente a dos grupos, o distribuía su periodo de formación en dos clases, una con el grupo de diversificación donde desarrolla los contenidos de las asignaturas troncales agrupadas en dos bloques o áreas (socio-lingüística y científico-tecnológica) y otra correspondiente a su grupo de referencia, donde cursa Educación Física, Plástica y Visual, Música, Religión o Alternativa. La idea de esta dicotomía es evitar al máximo la desconexión de los estudiantes con su grupo, a la vez que se les facilita un currículum adaptado a sus características. Los resultados en la provincia de Sevilla arrojan datos favorables sobre esta medida, ya que en un estudio realizado por Navarro (2006) sobre la opinión de 142 estudiantes que cursaban P.D.C. en 16 I.E.S. de la capital, el 93,7\% considera que aprende mejor en este grupo, frente al 4,9\%, que opta por su grupo clase; el 45,8\% considera que lo mejor del P.D.C. es que, al ser pocos, les atienden mejor y, sólo el 3,5\%, ve como negativo estar separados de sus compañeros. Aunque pertenezca a otro contexto, el estudio realizado por Aramendi, Vega y Santiago (2011) en el País Vasco, también muestra que el alumnado que cursaba P.D.C. estaba satisfecho, aunque menos que los que cursaban Programas de Cualificación Profesional Inicial (PCPI), que tenían un carácter más práctico, y con expectativas altas para obtener el título (3,23 de media, siendo 4 el máximo posible).

En definitiva, son pocas las investigaciones realizadas sobre su repercusión en la Educación Secundaria, tuvo luces y sombras, sirvió para que muchos estudiantes que no tenían opción por la vía ordinaria alcanzaran el Título de Graduado en Educación Secundaria, aunque muchas veces sin las herramientas necesarias para continuar con éxito estudios posteriores:

"El programa de Diversificación Curricular es otra herramienta disponible en el segundo ciclo de la ESO. Un $28 \%$ de estos expedientes, al menos, continúa su formación en ciclos post-obligatorios aunque fracasen en ellos, llegando casi a un tercio si incluimos los casos del Bachillerato a distancia. Esto indica que esta herramienta tiene más éxito que cualquiera de las anteriores, y que la atención diversificada de los alumnos es efectiva". (Mena, Fernández y Rivière, 2010, 130).

Los P.D.C. continuaron aplicándose tras el cambio legislativo de 2006, la Ley Orgánica de Educación, los mantuvo con la misma estructura, sólo anticipó de forma unánime el curso de aplicación desde $3^{\circ}$ de E.S.O. (art. 27), ya que anteriormente era necesario solicitar y justificar su necesidad por parte de cada centro para su desarrollo. Sus herederos directos son los Programas de Mejora del Aprendizaje y del Rendimiento (PMAR), que son propuestos por la Ley Orgánica para la 
Mejora Educativa (LOMCE) de 2013, aunque con algunas diferencias. La aprobación de esta Ley, no exenta de polémica, como la mayoría de las realizadas en España, supuso, según, Tarabini y Montes (2015), un punto de inflexión en la lucha contra el Abandono Escolar Prematuro (AEP), fundamentalmente acentuado en la Educación Secundaria Obligatoria, donde introduce los Programas de Mejora del Aprendizaje y del Rendimiento (PMAR), la Formación Profesional Básica (FPB) y la anticipación de los itinerarios hacia Bachillerato y Formación Profesional a $4^{\circ}$ de ESO, cuestiones que estos autores consideran más excluyentes que inclusivas, pero este no es el debate ahora.

El término Abandono Escolar Prematuro se utiliza para definir a las personas que han abandonado la enseñanza prematuramente y no siguen ningún tipo de educación o formación, incluyendo a la población de 18 a 24 años con unos estudios correspondientes a la Educación Secundaria Obligatoria o inferiores y que no sigue ningún tipo de enseñanza o formación (Adame y Salvá, 2010,187), y cuya reducción, hasta el $10 \%$, es uno de los objetivos europeos y españoles para la educación y formación en 2020 (MECD, 2013). En el año 2012, el abandono escolar se situaba en el $24,7 \%$ y pasó al $23,6 \%$ en el año 2013 , al $21,9 \%$ en 2014 y al $20 \%$ en 2015 . Por tanto, el abandono educativo temprano se ha reducido, situándose, durante el segundo trimestre, del año 2016, en el 19,7\%, siendo la cifra más baja contabilizada en nuestro país (MECD, 2016), pero alejada todavía de los objetivos para 2020.

Por Comunidades Autónomas, según la misma fuente, (ver gráfico 1) los porcentajes más bajos de abandono se producen en el País Vasco $(7,9 \%)$, Cantabria $(8,6 \%)$ y Navarra $(13,4 \%)$ y los más altos en Ceuta y Melilla con un 45,9\%, seguidas de Baleares con un 26,8\% y la región de Murcia con un 26,4\%.Sin embargo, en el segundo trimestre de 2015, las mejoras más significativas se producen en la Comunidad Valenciana (-1,2 puntos), Canarias (-3 puntos) y Andalucía (-1,8 puntos).

Figura 1

Evolución del abandono educativo temprano por Comunidades Autónomas

\begin{tabular}{|c|c|c|c|c|c|}
\hline Comunidades Autónomas & 2012 & 2013 & 2014 & 2015 & 2016 \\
\hline Andalucía & 28,8 & 28,7 & 27,7 & 24,9 & 23,1 \\
\hline Aragón & 20,4 & 18,9 & 18,4 & 19,5 & 19,1 \\
\hline Asturias (Principado de) & 19,8 & 19,1 & 13,6 & 16,8 & 16,6 \\
\hline Balears (Illes) & 28,9 & 29,8 & 32,1 & 26,7 & 26,8 \\
\hline Canarias & 28,0 & 27,5 & 23,8 & 21,9 & 18,9 \\
\hline Cantabria & 14,2 & 12,1 & 9,7 & 10,3 & 8,6 \\
\hline Castilla y León & 21,7 & 19,2 & 16,8 & 16,7 & 17,3 \\
\hline Castilla - La Mancha & 27,5 & 27,4 & 22,2 & 20,8 & 23,2 \\
\hline Cataluña & 24,2 & 24,7 & 22,2 & 18,9 & 18,0 \\
\hline Comunitat Valenciana & 25,9 & 21,7 & 23,4 & 21,4 & 20,2 \\
\hline Extremadura & 32,6 & 29,2 & 22,9 & 24,5 & 20,4 \\
\hline Galicia & 22,7 & 20,2 & 18,5 & 17,0 & 15,2 \\
\hline Madrid (Comunidad de) & 21,5 & 19,7 & 18,3 & 15,6 & 14,6 \\
\hline Murcia (Región de) & 26,9 & 26,3 & 24,1 & 23,6 & 26,4 \\
\hline Navarra (Comunidad Foral de) & 13,0 & 12,9 & 11,8 & 10,8 & 13,4 \\
\hline País Vasco & 12,4 & 9,9 & 9,4 & 9,7 & 7,9 \\
\hline Rioja (La) & 24,3 & 21,7 & 21,1 & 21,5 & 17,8 \\
\hline Ceuta & 38,5 & 33,5 & 29,5 & 29,8 & 21,5 \\
\hline Melilla & 32,6 & 33,1 & 19,6 & 24,1 & 24,4 \\
\hline
\end{tabular}

Fuente: adaptado de MECD 2016. 
Las causas pueden ser muchas, tantas como las que motivan el abandono, cuyos detalles recogen exhaustivamente autores como Mena, Fernández y Riviere (2010), pues los cambios legislativos de 2013 no llegaron a aplicarse en Secundaria hasta el curso 2015-2016, en $3^{\circ}$, y, 2016-2017, en $2^{\circ}$ de ESO, y, todos sabemos que relacionar un problema multifactorial con una sola causa, sería cuanto menos incauto. Las investigaciones más recientes ya han asumido este carácter, tratando de hacer propuestas que no sólo impliquen a los centros educativos, sino a toda la comunidad (Parrilla et. al, 2017), de forma inclusiva y preventiva. Además, vienen haciéndose eco de la necesidad de identificar factores de exclusión, como ya hicieran otros autores en Inglaterra (Booth y Ainscow, 2000), con objeto de promover mejoras desde la reflexión y acción conjunta, basándose en tres dimensiones: cultura, política y práctica educativa, pero los cambios a gran escala son lentos y costosos.

En un plano más operativo, y centrado en las características de los estudiantes, podemos considerar que el alumnado en riesgo de exclusión o vulnerable, es aquel que (Vallejo y Bolarín, 2009, 144, basado en Nieto, 2004):

- Manifiesta dificultades propiamente cognitivas y en rendimiento académico.

- Tiende a calificarse como alumnos social y escolarmente inadaptados, con problemas de relación y de comportamiento.

- Pertenece y comparte una determinada cultura de iguales, combinada de formas peculiares con la cultura familiar, en la que la escuela y el estudio están muy devaluados.

- Ha ido pasando de curso "por los pelos", quizás, entre otras razones, porque no son conflictivos respecto al orden establecido y valorado por la escuela, así como aquellos otros que, según el argot, han pasado de curso o de ciclo "por imperativo legal".

- Se esfuerza, y que trabaja, pero que "su inteligencia no les da para más".

- Aunque se reconoce que serían capaces e inteligentes, si quisieran, se han "desenganchado" de la escuela, han tenido una historia escolar marcada por relaciones problemáticas con algunos de sus profesores o compañeros, cristalizando todo ello con el tiempo en un conjunto de disposiciones, intereses y horizontes vitales entre los que lo escolar y lo académico ocupa, quizás, un lugar más que secundario.

De esta tipología de estudiantes, obtenidas a partir de la visión del profesorado que participó en P.D.C. y P.C.P.I. en la región de Murcia durante los primeros años del milenio, no todos cumplirían los requisitos propuestos por la administración para integrarse en un PMAR, como veremos posteriormente.

Y para terminar con otro de los principios en los que se basan estos Programas, definimos los agrupamientos flexibles como una medida de atención a la diversidad que consiste en un "tipo de agrupación del alumnado entre los diferentes grupos de un mismo nivel o curso, rompiendo el grupo de referencia y estableciendo diferentes grupos en función de los distintos niveles de competencia curricular" (Ortega, 2010, 3). Esta modalidad de agrupamiento tiene como objetivo favorecer la atención a las diferencias individuales desde la adaptación del proceso de enseñanzaaprendizaje a los intereses y ritmos de aprendizaje de cada alumno (Fernández, 2006), aunque sería una, entre otras muchas, para lograr una escuela inclusiva.

\section{Los Programas para la Mejora del Aprendizaje y del Rendimiento}

El artículo 27 de la LOE, que trata sobre los Programas de Diversificación Curricular (PDC), es sustituido por el artículo 18 de la LOMCE, que introduce los Programas de Mejora del Aprendizaje y Rendimiento (PMAR) con una breve descripción sobre su duración, procedimientos y requisitos de acceso del alumnado. El desarrollo de los PMAR se recoge en el artículo 19 del Real Decreto 1105/2014, de 26 de diciembre, por el que se establece el currículo básico de la Educación Secundaria Obligatoria y del Bachillerato, de donde resaltamos las siguientes características: 
- Se desarrolla a partir de $2^{\circ}$ curso de la ESO y tiene una duración de dos años, excepcionalmente aquellos alumnos y alumnas que, habiendo cursado $3^{\circ}$ de Educación Secundaria Obligatoria, no estén en condiciones de promocionar a $4^{\circ}$, podrán incorporarse a un Programa de mejora del aprendizaje y del rendimiento para repetir 3er curso.

- Utilizará una metodología específica, a través de la organización de contenidos, actividades prácticas y, en su caso, de materias diferentes a las establecidas con carácter general.

- Tiene la finalidad de que los alumnos y alumnas puedan cursar $4^{\circ}$ curso por la vía ordinaria y obtengan el título de Graduado en Educación Secundaria Obligatoria.

- Se dirige a aquellos alumnos y alumnas que presenten dificultades relevantes de aprendizaje no imputables a falta de estudio o esfuerzo. También para el alumnado con "discapacidad".

- El equipo docente podrá proponer a los padres, madres o tutores legales la incorporación a un Programa de mejora del aprendizaje y del rendimiento de aquellos alumnos y alumnas que hayan repetido, al menos, un curso en cualquier etapa y que, una vez cursado $1^{\circ}$ de ESO, no estén en condiciones de promocionar a $2^{\circ}$ curso, o que una vez cursado $2^{\circ}$, no estén en condiciones de promocionar a $3^{\circ}$.

- En todo caso, su incorporación requerirá la evaluación, tanto académica como psicopedagógica, y la intervención de la Administración educativa en los términos que ésta establezca; y se realizará una vez oídos los propios alumnos o alumnas y sus padres, madres o tutores legales.

- Se potenciará la acción tutorial como recurso educativo que pueda contribuir, de una manera especial, a subsanar las dificultades de aprendizaje y a atender las necesidades educativas del alumnado.

- La evaluación tendrá como referente fundamental las competencias y los objetivos de la Educación Secundaria Obligatoria, así como los criterios de evaluación y los estándares de aprendizaje evaluables.

La ley establece dos modalidades para su desarrollo, una con realización del Programa dentro del aula ordinaria, y otra donde se comparten asignaturas específicas, en un grupo, con otras asignaturas en el grupo ordinario:

- Organización de forma integrada: el alumnado del programa cursará en grupos ordinarios todas las materias del $2^{\circ}$ y $3 \mathrm{er}$ curso, si bien éstas serán objeto de una propuesta curricular específica, en la que los contenidos podrán agruparse por ámbitos de conocimiento, por proyectos interdisciplinares o por áreas de conocimiento, y, que requerirá, en todo caso, de una orientación metodológica adaptada.

- Programa organizado por materias diferentes a las establecidas con carácter general: se podrán establecer al menos tres ámbitos específicos, compuestos por los siguientes elementos formativos:

1. Ámbito de carácter lingüístico y social, que incluirá, al menos, las materias troncales: Lengua Castellana y Literatura, Geografía e Historia, y Lengua Cooficial y Literatura (si la hubiere).

2. Ámbito de carácter científico y matemático, que incluirá, al menos, las materias troncales: Biología y Geología, Física y Química, y Matemáticas.

3. Ámbito de lenguas extranjeras: en esta modalidad, se crearán grupos específicos para el alumnado que siga estos programas, el cual tendrá, además, un grupo de referencia con el que cursará las materias no pertenecientes al bloque de asignaturas troncales.

No obstante, en la mayoría de los centros, se ha optado por la opción "b", bien por su similitud con los PDC, o por facilitar la organización y la atención más personalizada en grupos reducidos.

A continuación, presentamos un cuadro donde se observan las principales diferencias entre los Programas de Diversificación Curricular y los de Mejora del Aprendizaje y del Rendimiento. 
Tabla 1

Diferencias entre el Programa de Diversificación Curricular y el Programa de Mejora del Aprendizaje y el Rendimiento

PDC

PMAR

Dirigido a aquellos alumnos que repiten el $2^{\circ}$ o $3^{\circ}$ curso de la ESO y que no están en condiciones para promocionar al siguiente, o bien, aquel alumnado que ha sido objeto de otras medidas de atención a la diversidad en cursos anteriores, incluida, en su caso, la adaptación curricular significativa.

Se imparte en el $3^{\circ}$ (sólo con solicitud del centro en

LOGSE, en LOE se amplía desde $3^{\circ}$ ) y $4^{\circ}$ curso de Se imparte en el $2^{\circ}$ y $3^{\circ}$ curso de ESO
Dirigido a aquellos alumnos que presentan dificultades relevantes de aprendizaje que no se imputen a una falta de estudio. Y alumnado con "discapacidad".

Metodología específica través de una organización de contenidos, actividades prácticas y, en su caso, de materias, diferente a la establecida con carácter general.

Programa de diversificación curricular que incluye en su estructura los siguientes elementos:

- Ámbito lingüístico y social

- Ámbito científico-tecnológico

- Tres materias obligatorias u opcionales

- Tutorías grupo clase

- Materias optativas

Tutoría específica con el orientador/a en el grupo de diversificación
Programas organizados de manera integrada y por materias diferentes.

Organización de manera integrada: el alumnado cursará en grupos ordinarios todas las materias del $2^{\circ}$ y $3^{\circ}$ curso, siendo objeto de una propuesta curricular específica

Organización por materias diferentes:

- Ámbito de carácter lingüístico y social

- Ámbito de carácter científico y matemático

- Ámbito de lenguas extranjeras.

Potencia la acción tutorial

Fuente: elaboración propia.

La principal diferencia que podemos resaltar es el inicio y finalización del programa. El PMAR empieza un año antes ( $2^{\circ}$ curso), acabando en $3^{\circ}$, mientras que el Programa de Diversificación Curricular comienza en $3^{\circ}$, acabando en $4^{\circ}$. El problema fundamental que suscita el PMAR es, si los estudiantes, que han estado en el programa durante ambos cursos, serán capaces de titular en $4^{\circ}$ cuando pasan a la vía ordinaria, donde se cambia de nuevo totalmente la organización del currículum y la atención que reciben. Según la legislación, el objetivo de este Programa de Mejora del Aprendizaje y del Rendimiento, no es el hecho de llegar a obtener el título en Educación Secundaria Obligatoria, sino, conseguir que los alumnos y alumnas lleguen a cursar $4^{\circ}$ de ESO dentro del grupo ordinario de clase. Tiene un carácter reparador más que finalista, pero es contradictorio que los estudiantes que, durante dos cursos, han recibido un nivel más adaptado del currículum a sus características, luego vuelvan a la vía que les ha hecho fracasar.

En cuanto a las materias, en teoría, la LOE integra menos ámbitos que la LOMCE, aunque en la práctica, la inclusión de un idioma extranjero se podía solicitar, en función de las características del grupo de estudiantes.

La evaluación en ambos programas es equiparable a la de la ESO, con criterios específicos dentro de cada programa. Por último, refiriéndonos a la promoción, en el PDC los estudiantes repetían en el programa si no estaban en condiciones de titular. Sin embargo, actualmente los estudiantes que hayan cursado PMAR y pasen a $4^{\circ}$ repetirán en el curso ordinario, ya que, como hemos comentado, en $4^{\circ}$ no se benefician de dicho programa. Sólo en casos excepcionales se podrá establecer un grupo de $3^{\circ}$ de PMAR para repetir $3^{\circ}$ de ESO, dentro del programa. 
Concretando las características específicas de los PMAR, cada ámbito tiene un número de horas a la semana: el ámbito lingüístico-social dedica 7 horas lectivas, el ámbito científico-matemático ocupa 9 horas y el ámbito de lenguas extranjeras 3 horas semanales. También tienen una hora de tutoría específica, generalmente a cargo del Dpto. de Orientación. Las asignaturas troncales, organizadas por ámbitos, son impartidas en grupos específicos en los que se encuentran sólo los estudiantes pertenecientes al Programa de Mejora del Aprendizaje y del Rendimiento, que oscila entre 8 y 15. El resto de asignaturas se imparten en el grupo de referencia integrado por todos los estudiantes del curso. A la semana se imparten un total de 30 horas lectivas, contando las horas que ocupan las asignaturas organizadas por ámbitos y las asignaturas que se imparten con el resto del grupo ordinario. En este grupo se imparte Música, Educación Plástica y Visual, Educación Física y una materia optativa, con 2 horas lectivas semanales cada una, Religión o Alternativa y Tutoría, durante una hora cada cual.

La evaluación que se establece para el alumnado que cursa la ESO dentro de este Programa educativo, es decidida por el conjunto del Equipo Docente, en función de la consecución de los objetivos y competencias fijados al comienzo del curso. Se ajusta a los contenidos que lo componen, siendo la misma continua, formativa e integradora. Será adaptada a aquellos estudiantes que tengan Necesidades Educativas Especiales.

Para poder promocionar al curso siguiente, el estudiante no puede tener "no superados" 2 ó 3 ámbitos. Eso sí, si el Equipo Docente considera que los ámbitos "no superados", no impiden que éste desarrolle con éxito el siguiente curso, promocionará. Una vez finalizado el curso y realizada la evaluación, los padres, madres o tutores legales recibirán las notas con un consejo del orientador acerca de los objetivos y competencias alcanzadas por su hijo/a. También, en caso necesario, será transmitida una propuesta por parte del mismo orientador/a, como, por ejemplo: acceso al Programa de Mejora del Aprendizaje y del Rendimiento o acceso a la Formación Profesional Básica.

Si el estudiante supera el curso de $2^{\circ}$ y $3^{\circ}$ con éxito, deberá elegir entre dos itinerarios educativos al llegar al curso de $4^{\circ}$ con el resto de clase ordinaria. Estos itinerarios son: enseñanzas académicas, para el acceso a Bachillerato, y enseñanzas aplicadas, para el acceso a Formación Profesional.

\section{Planteamiento del estudio y objetivos}

Este estudio supone un acercamiento a la realidad de los diferentes agentes que participan en los PMAR en algunos centros de la provincia de Sevilla, con el objetivo de conocer de primera mano su implementación en los centros de Secundaria, ya que, al tratarse de una medida relativamente reciente, son pocas las investigaciones realizadas sobre los mismos. Surge de las propuestas realizadas a varias estudiantes del Grado en Pedagogía para la realización de sus Trabajos Fin de Grado, con un doble objetivo, iniciarlas en su carrera como futuras investigadoras, fomentando un espíritu crítico, identificando la realidad de los centros y comparándola con la legislación vigente, además de formarse sobre las diversas medidas de atención a la diversidad que existen en esta etapa, por si optan ser Orientadoras de Secundaria.

Para que se cumplieran estos objetivos, cada estudiante, con el apoyo docente plantea sus propios problemas de investigación y los instrumentos que considera adecuados, que, posteriormente, aplican en los centros con más facilidad de acceso. Esto provoca que contemos con diferentes instrumentos; concretamente cuestionarios y entrevistas estructuradas, de diferente longitud y diferentes temas, y, que la muestra sea incidental, respondiendo a la proximidad de las estudiantes con los centros y la facilidad de acceso a los mismos.

Los objetivos, en cambio, sí son comunes en la mayoría de los casos, pues se fijaron desde el principio con la docente en líneas generales, siendo éstos:

- Conocer cómo se elaboran los PMAR, quiénes participan y qué características tienen en diversos Institutos de Educación Secundaria. 
- Identificar cómo se realiza el proceso de selección del alumnado para su inclusión en el PMAR, así como la actitud de las familias cuando se les plantea.

- Establecer si el perfil del alumnado derivado a los PMAR coincide con el propuesto por la legislación

- Observar los cambios fundamentales que se introducen en los currículums de los ámbitos del PMAR.

- Saber el grado de satisfacción que tienen los estudiantes que cursan el Programa, el profesorado y orientadores.

- Identificar las expectativas del alumnado, el profesorado y los orientadores respecto a la continuidad en estudios superiores.

\section{Metodología}

Dado el carácter de la investigación, como un acercamiento a la realidad de los centros de Secundaria que imparten PMAR en la provincia de Sevilla, que surge de los trabajos realizados por el alumnado, la metodología es mixta basada en una combinación de las metodologías cualitativa y cuantitativa. Se trata pues de pequeños estudios de campo en varios institutos, en los que posteriormente, mediante un análisis documental, identificaremos rasgos comunes y diferenciales entre los mismos, tratando de arrojar luz sobre cómo valoran los diferentes participantes los PMAR y dar respuesta a los objetivos planteados.

\subsection{Muestra}

Como adelantamos, la muestra es incidental y no responde a ningún criterio previo, sino a la facilidad de acceso al campo de las estudiantes, no obstante, podemos identificar varias zonas de análisis: una perteneciente a la Sierra Norte de Sevilla (IES "ROD” Y "HER"), que se sitúa a unos $125 \mathrm{Km}$ de la capital, otra de la zona, situada a $21 \mathrm{Km}$ de la capital de provincia, Mairena del Alcor (IES “ALC" y "MAI") y, por último, un pueblo muy próximo a la capital, que casi es considerado un barrio de la misma, La Algaba, a sólo $17 \mathrm{Km}$ (IES "MAC" y "TOG"). En todos los IES han participado en las investigaciones el alumnado, profesorado de los ámbitos y jefes/as de los departamentos de orientación, obteniendo la siguiente muestra: 96 estudiantes, 19 profesores/as de los ámbitos y 6 orientadores/as.

Tabla 2

Distribución de la muestra por centros y grupos

\begin{tabular}{ccccc}
\hline IES & Alumnado & Profesorado & Orientadores & Grupos \\
\hline "ROD" & 8 & 4 & 1 & $3^{\circ} \mathrm{ESO}$ \\
"HER" & 6 & 2 & 1 & $3^{\circ} \mathrm{ESO}$ \\
"MAC" & 17 & 2 & 1 & $2^{\circ}$ y $3^{\circ} \mathrm{ESO}$ \\
"ALC" & 13 & 2 & 1 & $2^{\circ}$ y $3^{\circ} \mathrm{ESO}$ \\
"MAI" & 23 & 4 & 1 & $2^{\circ}$ y $3^{\circ} \mathrm{ESO}$ \\
TOTAL: 6 centros & 29 & 5 & 1 & $2^{\circ}$ y $3^{\circ} \mathrm{ESO}$ \\
\hline
\end{tabular}

Fuente: elaboración propia.

Atendiendo al sexo encontramos 5 orientadoras $(83,3 \%)$ y un orientador; 16 profesoras $(84,2 \%)$ y 3 profesores; 51 alumnas $(53,1 \%)$ y 45 alumnos. Las edades del personal docente oscilan entre los 35 y 55 años, y, entre los estudiantes, la media se sitúa en 15 años de edad. 


\subsection{Instrumentos utilizados}

Se han utilizado dos tipos de instrumentos para la recogida de datos, cuestionarios tipo Likert para el alumnado y el profesorado y entrevistas para los/as orientadores/as. Las cuestiones de la entrevista han sido adaptadas de Castro y Corujo (2006) y los cuestionarios, al no encontrar ninguno adecuado para esta investigación, han sido elaborados en función de los objetivos de la misma, y aplicados, tras una validación de expertos. Los cuestionarios permiten aplicarse en poco tiempo, para no interferir mucho en las clases, y extraer conclusiones de forma rápida (Herrera, 2002), en nuestro caso se han diseñado dos cuestionarios diferentes para alumnado y profesorado, pero su elaboración y forma de aplicación es la misma.

Tienen una primera parte donde se explica el objetivo y se preguntan varios datos personales, y, una segunda parte con una serie de afirmaciones donde el alumnado y el profesorado han de mostrar su grado de acuerdo, siguiendo una escala tipo Likert, siendo, 1 "mínimo" y 5 "máximo acuerdo".

Los ítems del cuestionario para el profesorado y preguntas de la entrevista a los orientadores se agrupan en temas tales como: características del programa de mejora; perfil del alumnado que lo cursa y cambios observados tras su incorporación al mismo; organización, metodología y evaluación; reacciones de las familias ante la propuesta de inclusión de sus hijos e hijas en un PMAR; y expectativas de futuro respecto a los estudiantes.

Los temas de los cuestionarios para el alumnado son: el nivel de exigencia del programa, sus relaciones con el profesorado y el alumnado, los contenidos, las asignaturas y metodología, su rendimiento y sus expectativas futuras. En todos los cuestionarios y entrevistas se incluye una pregunta abierta donde se pide que enumeren las ventajas e inconvenientes del Programa, así como sugerencias de mejora. La aplicación de los mismos se realiza de forma directa en cada centro, los dos primeros durante el segundo trimestre del curso 2015-2016 y los cuatro siguientes en la primavera de 2016-2017.

\subsection{Análisis}

Se realizaron inicialmente dos tipos de análisis, uno cuantitativo, tras el vaciado de los cuestionarios, con el programa SPSS 23.0, y otro cualitativo de las entrevistas realizadas. Posteriormente, y, para dar una visión global de los resultados obtenidos, se ha realizado un análisis documental de los cuestionarios y las entrevistas, así como de los análisis previos, entre tres investigadoras, identificando y agrupando las preguntas que respondieran a los objetivos propuestos. Se puede definir el análisis documental como la operación, o conjunto de operaciones, tendentes a representar el contenido de un documento bajo una forma diferente de la suya original a fin de facilitar su consulta o localización en un estudio ulterior. (Andreú, 2000, 9). Tras el análisis, se establecieron las siguientes categorías y subcategorías: 1. Elaboración Programa, 1.1. Cómo, 1.2. Quién, 1.3. Características; 2. Selección Alumnado, 2.1. Proceso, 2.2. Actitud Familia, 2.3. Actitud Alumnado; 3. Perfil Alumnado; 4. Cambios Currículum, 4.1. Objetivos, 4.2. Contenidos, 4.3. Metodología, 4.4. Recursos, 4.5. Evaluación; 5. Evaluación Programa, 5.1. Grado Satisfacción, 5.2. Expectativas. Las categorías 1, 2, 3 y 4, corresponden con los mismos objetivos, y la 5 persigue los objetivos 5 y 6 . A continuación presentamos los resultados del análisis documental realizado.

\section{Resultados}

Para presentar los resultados obtenidos, vamos a utilizar los objetivos planteados, intentando dar respuesta a cada uno de ellos, teniendo en cuenta las opiniones de los agentes implicados. La mayoría de preguntas han sido respondidas por todos (alumnado, profesorado y orientadores), lo que nos permitirá hacer una valoración más completa de la realidad observada, y, otras, sólo por dos o uno de ellos, atendiendo a los objetivos perseguidos; en la tabla 3 se muestra la correspondencia entre objetivos y fuentes de información. 
Tabla 3

Relación entre objetivos y fuentes de información

Agentes o fuentes de información

\begin{tabular}{cccc}
\hline Objetivo & Alumnado & Profesorado & Orientadores \\
1 & & $\mathrm{X}$ & $\mathrm{X}$ \\
2 & $\mathrm{X}$ & $\mathrm{X}$ & $\mathrm{X}$ \\
3 & $\mathrm{X}$ & $\mathrm{X}$ & $\mathrm{X}$ \\
4 & $\mathrm{X}$ & $\mathrm{X}$ & $\mathrm{X}$ \\
5 & $\mathrm{X}$ & & \\
6 & $\mathrm{X}$ & $\mathrm{X}$ & $\mathrm{X}$ \\
\hline
\end{tabular}

Fuente: elaboración propia.

El primer objetivo, conocer cómo se elaboran los PMAR, quiénes participan y qué características tienen en diversos Institutos de Educación Secundaria, será respondido por el profesorado y los orientadores. En todos los casos se ha optado por organizarlos en materias diferentes a las establecidas con carácter general, en ámbitos y grupos específicos, es decir, la opción "b" del Real Decreto 1105/2014. Por ejemplo, en el IES “ROD” el Programa Educativo en general tiene una duración de 15 horas de clase semanales, distribuidas en torno a 2 ámbitos; 7 horas son dedicadas al ámbito de científico-matemático, en el que se incluyen las materias de Matemáticas, Biología, Física y Química. Otras 8 horas son dedicadas al ámbito socio-lingüístico, en el que se incluyen las materias de Lengua Castellana y Literatura, y Ciencias Sociales. En el IES "HER" no se incluye inglés, lo que dificulta su continuidad en $4^{\circ}$. En todos los centros se elabora el programa atendiendo a las características del alumnado, siguiendo los procedimientos fijados por la legislación; en su confección participan los profesores de los ámbitos, así como el Dpto. de Orientación y los tutores de los estudiantes de sus grupos de referencia.

En segundo lugar, queremos identificar cómo se realiza el proceso de selección del alumnado para su inclusión en el PMAR, así como la actitud de las familias y del alumnado cuando se les plantea. Para responder a la primera parte del objetivo, ¿cómo se realiza el proceso de selección del alumnado?, analizamos las respuestas del profesorado y los orientadores, observando un alto grado de acuerdo respecto al procedimiento, ya que éste está determinado claramente en la legislación. A modo de resumen, en todos los centros estudiados, por consenso del Equipo Educativo, se propone para su inclusión al PMAR a una serie de alumnos y alumnas, a los que el Dpto. de Orientación realizará una evaluación psicopedagógica con pruebas orales y escritas, mediante test o escalas de autoestima y personalidad, así como entrevistas para conocer sus intereses, ya que un requisito es tener motivación y actitud positiva. Por otro lado, el orientador, también se entrevista con las familias para explicarles esta medida y obtener su consentimiento, tanto para la evaluación, como para la inclusión en el PMAR, ya que son requisitos indispensables, según la legislación.

En el IES "MAC", un 11,1\% del profesorado, considera que el proceso que se sigue para la selección del alumnado, no se adecua al establecido por la LOMCE, aunque, al tratarse de un cuestionario cerrado, no podemos responder a lo que se referían.

Respecto a la opinión de las familias, encontramos un acuerdo casi mayoritario a cursar el PMAR, aunque varias orientadoras expresan algunas discrepancias:

"existen algunas ocasiones en las que los padres y madres no se encuentran del todo convencidos con este cambio" (Orientadora del IES “HER”).

"hay un cierto rechazo inicial hacia el programa porque llegan a pensar que sus hijos e hijas van a perder expectativas académicas al cursarlo" (Orientadora del IES “ALC”).

"la mayoría del alumnado y las familias aceptan el programa, pero muchas de éstas se lo piensan, y, sólo utilizan esta vía como último recurso, prefiriendo otras medidas de atención o la repetición" (Orientadora IES “MAC”). 
En cambio, el alumnado lo contradice, ya que un $70 \%$ ha dicho que sus familias están "muy contentas" o "bastante contentas" de que cursen PMAR, un 23,33\% responden que sus familias están "poco contentas" y un 6,66\% que "no están contentas" o "muy poco", en los IES "MAC" y "TOG"; y el 100\% de los estudiantes pertenecientes a los IES "ROD" y "HER", afirman que sus familias están de acuerdo con el hecho de que ellos estén realizando el curso dentro de este Programa.

Para conocer la actitud del alumnado cuando se les plantea acceder a un PMAR, encontramos también percepciones diferentes.

Si le preguntamos a los profesores, el 62,5\% de ellos, están "de acuerdo" o "completamente de acuerdo" con que la mayoría de alumnado desea entrar en PMAR una vez que el centro le ha hecho la propuesta de incorporación, frente al 37,5\%, que están "en desacuerdo" o "poco de acuerdo" con tal afirmación.

Los orientadores afirman que normalmente reaccionan bien, aunque se observan algunas reacciones diferentes, por ejemplo, en los IES "ALC" y "MAI", las orientadoras coinciden, afirmando que, normalmente el alumnado ve el programa como algo positivo y llega a encontrar una motivación en el mismo que le impulsa a seguir intentándolo, y, en el IES “TOG", la orientadora explica que hay alumnado que se alegra porque mejoran sus calificaciones, y otros, ante la propuesta, mejoran su rendimiento para no entrar en el programa. En ambos casos produciría un efecto positivo.

Para establecer si el perfil del alumnado derivado a los PMAR coincide con el propuesto por la legislación, encontramos que todos han repetido algún curso $\left(1^{\circ}, 2^{\circ}\right.$ o $3^{\circ}$ de ESO o alguno de Educación Primaria también, con un elevado número de asignaturas suspensas, entre 6 y 10), excepto en un I.E.S. de La Algaba, donde un estudiante afirma que no ha repetido ningún curso. En el IES "ALC", se consideran posibles candidatos aquellos estudiantes que tienen, como mínimo, 3 asignaturas pendientes en el primer trimestre y hayan repetido anteriormente. En el IES "MAI", su orientadora remarca otro de los requisitos de la ley, que tienen muy en cuenta: "el proceso de selección del alumnado a PMAR se realiza mediante una reunión con el Equipo Directivo para detectar a aquel alumnado que presenta dificultades de aprendizaje, que no son debidas a una falta de estudio o esfuerzo, sino que realmente son estudiantes a los que les cuesta bastante avanzar para conseguir los objetivos de la etapa en la que se encuentran”.

En cuanto a las dificultades que el alumnado presenta, no hay un acuerdo generalizado entre los orientadores, así, en el IES “ALC", se considera que la principal dificultad de aprendizaje en el alumnado del PMAR es la capacidad intelectual límite de varios estudiantes; en cambio, la orientadora del IES "MAI" considera que las dificultades de aprendizaje van más dirigidas a la competencia matemática, comprensión oral y escrita y, sobre todo, a los malos hábitos de estudios.

Por su parte, el alumnado de los IES "MAC" y "TOG" afirma tener "bastante" y "mucha" dificultad para concentrarse y estudiar (40\%), un 33,33\% responde que tiene "poca dificultad" y un $16,67 \%$, que "no tiene" o tiene "muy poca" dificultad. Por tanto, la mayoría no se ajustaría al perfil propuesto por la legislación para cursar el programa. Por último, observamos discrepancias del profesorado en estos centros, ya que sólo el 27,5\% está "de acuerdo" o "completamente de acuerdo" con que al PMAR siempre accede alumnado con dificultades relevantes de aprendizaje, no imputables a falta de estudio o esfuerzo, como puede observarse en el gráfico 2. 


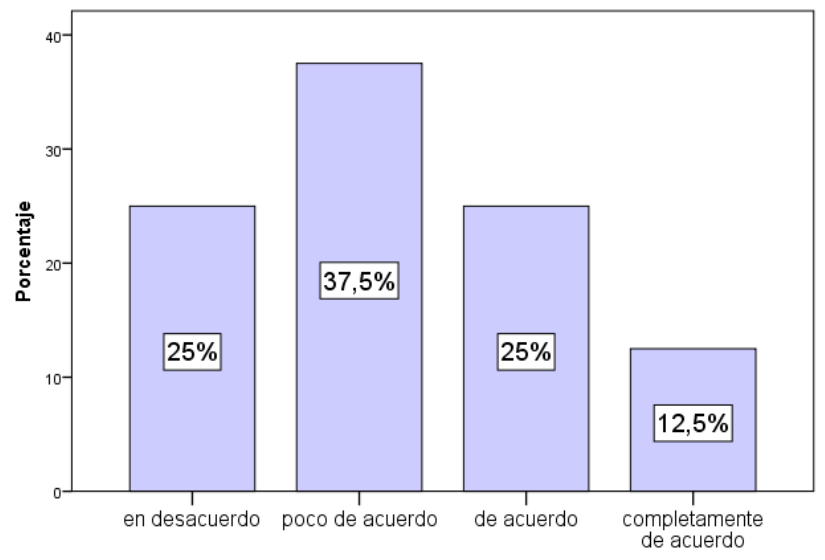

Fuente: elaboración propia.

El cuarto objetivo se centra en observar los cambios realizados en los diferentes elementos del currículum de los ámbitos del PMAR, para evitar un desarrollo amplio, vamos a describir las características encontradas en cada elemento:

- Los objetivos se ajustan a los criterios establecidos para la ESO a nivel general, aunque se refuerzan aquellos que son básicos y necesarios para el perfil del alumnado, incluyendo la autoestima, los hábitos y técnicas de estudio; comprensión y expresión oral y escrita, y, resolución de problemas.

- Los contenidos, igualmente se ajustan a los establecidos para la ESO, aunque agrupados por ámbitos, no obstante, el 88,8\% del profesorado encuestado considera que las asignaturas del programa," casi siempre" o "siempre" se adaptan a las necesidades del alumnado que lo cursa. Si no fuera así, perdería su carácter de medida de atención a la diversidad. Los estudiantes también lo consideran así en el $80,7 \%$ de los casos, y casi la mitad de ellos, opinan que las asignaturas son más fáciles que en la ESO.

- La metodología es la que experimenta más cambios, principalmente porque, al ser grupos reducidos, y tener más horas con los mismos profesores, permite realizar más actividades en grupos y parejas, que es donde el alumnado reconoce aprender más ( $90 \%$ del IES "ROD”). El profesorado coincide en que se desarrolla una metodología más personalizada, pausada y adaptada a lo que se esté viendo en el momento. "Las explicaciones no se dan de manera más detenida, sino más detallada; como al alumnado le cuesta más trabajo, se dan explicaciones más cortas y cercanas a sus intereses, dándoles más tiempo para asimilarlas" (profesor de ámbito). A diario se realiza trabajo en clase, actividades motivadoras, prácticas para conseguir los objetivos fijados, refuerzo de técnicas y objetivos básicos, trabajo en las diferentes materias por bloques relacionados, revisión de la libreta, realización de actividades, por parte de los estudiantes, en la pizarra; es decir, metodología práctica, dinámica y que fomenta la realización de pequeños trabajos de investigación.

- Se hace uso de recursos tecnológicos en algunas clases (ordenadores portátiles y personales que hay en las clases ordinarias, para buscar y seleccionar información, elaborar documentos), también se hace uso de la pizarra digital, se visionan vídeos, exponen trabajos y actividades con recursos audiovisuales, esto facilita la motivación y aumenta el interés del alumnado.

- La evaluación se realiza de forma continua, se valora la actitud y el esfuerzo, así como el trabajo diario. Como instrumentos de evaluación encontramos exámenes escritos, exámenes orales, trabajos obligatorios y/o voluntarios, actividades realizadas a diario en clase, comportamiento y asistencia a clase. En los exámenes, hay ocasiones en las que los discentes pueden hacer uso de apuntes o el vocabulario de clase, en función de la unidad que se esté viendo, según el profesorado, aunque los estudiantes lo desmienten. La evaluación -inicial, continua y final- es realizada por el Equipo Educativo. En cuanto a los criterios de 
evaluación, el orientador del IES "MAC" confirma que no son los mismos que en una clase ordinaria. Sin embargo, la orientadora del IES "TOG” dice que los criterios de evaluación son los mismos del nivel ordinario con algunas modificaciones en cuanto a porcentajes o instrumentos. El 87,5\% del profesorado de los IES "MAC" y “TOG” apoyan esta visión. Los estudiantes por su parte consideran que se les evalúa con justicia, sobre los contenidos que han visto en clase, y con más facilidades para aprobar que en la ESO.

El quinto objetivo es saber el grado de satisfacción que tienen los estudiantes que cursan el PMAR; para ello hemos contado sólo con su propia opinión a diferentes preguntas. Para facilitar la exposición de los resultados obtenidos, y no perder las aportaciones realizadas por el alumnado, agruparemos los mismos por poblaciones estudiadas.

En la mayoría de los casos encontramos un alto grado de satisfacción por diferentes motivos:

- De los estudiantes del IES "ROD", 5 (62,5\%) reconocen que prefieren estar en este Programa porque comprenden mejor el temario de las materias, reciben mayor ayuda, y, el ser menos en clase, les permite prestar mayor atención. Sólo dos chicas y un chico (37,5\%), prefieren "no estar" en este Programa Educativo, ya que prefieren estar con el resto de sus amigos, aunque reconocen que aquí aprenden más. El 75\% afirman que las expectativas que tenían acerca del Programa se han cumplido, y lo argumentan porque: hay menos estudiantes en clase (algo que ven como positivo), ahora aprueban, estudian más y les dan facilidades en el estudio, los temas les parecen más fáciles.

- En el IES "HER", sólo un alumno quisiera seguir en la ESO ordinaria porque "al salir de su clase se siente distinto"; los 5 restantes, (83,33\%), añaden a los motivos anteriores que el PMAR es más cómodo, al haber menos estudiantes, y dicen que en clase ordinaria hay mal ambiente, hay más ruido. Todos, a excepción de uno, reconocen estar contentos dentro de este Programa, argumentando que están contentos con los compañeros, es más divertido, y tienen buenos resultados; además, todos dicen que los profesores explican más. Uno de ellos también reconoce que está contento porque este programa es una oportunidad para llegar a tener el nivel educativo necesario. Sólo un alumno reconoce no estar contento realizando este Programa, aunque también señala que es porque no estudia.

- El 70\% del alumnado de los IES "MAC" " "TOG" piensa que el clima de la clase le ayuda a trabajar "bastante" o "mucho". Mientras que un $20 \%$ ha respondido que le ayuda "poco" y un $9,99 \%$ que "nada" o "muy poco". Respecto a la atención que reciben del profesorado, el $76,6 \%$ piensa que es "mucha" o "bastante"; el 86,67\% ha respondido que en este grupo se sienten, "mucho" o "bastante" más a gusto que en los grupos ordinarios; se sienten más valorados el 83,3\%, y el 86,67\% afirma que aprenden "mucho" o "bastante" más fácilmente en el programa y que explican mejor.

- El 98,1\% del alumnado de los IES “ALC" y "MAI" recomendaría siempre el programa a aquellos estudiantes que tuviesen dificultades de aprendizaje. El 59,51\% afirma que, desde que está en PMAR, le interesan más los estudios "casi siempre" o "siempre", frente a un 1,9\% que considera que nunca ha mostrado interés por los estudios, y el resto que sólo aumenta su interés "algunas veces", o de forma irregular (30,77\%). Cuando le preguntamos si el PMAR le ayuda a superar las dificultades de aprendizaje mostradas en el curso anterior, la mayoría del alumnado, concretamente el 78,85\%, responde "siempre" o "casi siempre".

Por último, queremos identificar las expectativas del alumnado, el profesorado y los orientadores respecto a la continuidad en estudios superiores, lo que nos servirá como evaluación indirecta de los programas y conocer si, realmente, cumplen con la función que pretenden las administraciones educativas. En este caso encontramos más diferencias entre las opiniones de los tres agentes. Incluiremos las aportaciones por poblaciones, continuando con el procedimiento del objetivo anterior.

- En el IES "ROD", el 87,5\% de los estudiantes pretende acceder a una Formación Profesional de Grado Medio (CFGM), una vez finalizada la ESO, y, sólo 1 se conforma con 
obtener la titulación de ESO y finalizar su formación. Sin embargo los profesores tienen opiniones más pesimistas, tres de ellos creen que disminuye la posibilidad de realizar estudios superiores una vez finalizada la Educación Secundaria Obligatoria debido al nivel con el que llegan al PMAR y, sólo 1 confía en sus posibilidades; en todos los casos recomendarían realizar estudios de Formación Profesional, aunque la Orientadora es más optimista o menos determinista con respecto a esto, afirmando que "el acceso de estos estudiantes a estudios superiores, como por ejemplo una Formación Profesional de Grado Medio, Bachillerato, o Universidad, depende de diversos aspectos". Pero por otro lado, es consciente de la dificultad: "hay que tener en cuenta que estos estudiantes han accedido a este Programa Educativo debido a sus dificultades de aprendizaje y sus fracasos educativos anteriores. Esto quiere decir que resulta complicado que consigan el Título de la ESO por ellos mismos en el curso ordinario, lo que impediría acceder a otro tipo de formación educativa después de la ESO". Con esto se establece que el acceso de estos estudiantes a este Programa Educativo disminuye, en parte, sus posibilidades para desarrollar después otro tipo de estudios.

- En el IES "HER" hay tres estudiantes que tienen, como única expectativa, conseguir el Título en Educación Secundaria Obligatoria; un alumno quiere acceder a una Formación Profesional de Grado Medio, y dos alumnas quieren acceder al Bachillerato. Los profesores tienen también opiniones dispares; uno, no cree que disminuyan las posibilidades de acceder a realizar estudios superiores, ya que, al acabar, tienen el Título en Educación Secundaria Obligatoria y los contenidos mínimos superados. El otro profesor afirma que disminuyen las posibilidades de acceder a Bachillerato, debido a que los estudiantes sólo han dado los contenidos mínimos de varias materias, y esto hace que las dificultades de realizar Bachillerato aumenten. Aun así, afirma que no disminuyen las posibilidades de acceder a una Formación Profesional de Grado Medio, siendo el PMAR una buena vía para acceder a esta formación académica. Por último, la Orientadora estima que, el estar en este Programa, disminuye la posibilidad de entrar en la Universidad debido a que no se tratan todos los contenidos. Sin embargo, considera que tienen opción de acceder a una Formación de Grado Medio, incluso cree que es la mejor opción, aunque reconoce que no es suficiente para todos los estudiantes, ya que, durante el curso académico, dos alumnos del curso de $3^{\circ}$ de PMAR, abandonaron esta medida de atención a la diversidad.

- En los centros "ALC" y "MAI" no se recogieron las opiniones del alumnado sobre este aspecto y las del profesorado se encuentran bastante repartidas: el 55,5\% del profesorado considera que, el PMAR "algunas" o "muy pocas" veces prepara al alumnado para cursar $4^{\circ}$ de ESO con posibilidades de éxito, mientras que el 44,4\% restante se divide entre las opciones, 1 "nunca" y 4 "casi siempre". Por lo que podemos decir que el PMAR, en poco más del 50\% de ocasiones, prepara al alumnado para cursar $4^{\circ}$ de ESO, con posibilidades de éxito. Las orientadoras de ambos centros consideran el PMAR como "solución al fracaso total o parcial", considerándolo como una solución al problema del fracaso, al intentar rescatar a aquel alumnado que en algún momento de la etapa se ha desenganchado del sistema, ofreciéndole una nueva oportunidad.

- En los centros "MAC" y "TOG", el 76,67\% de los estudiantes están, "bastante" o "muy seguros", de que van a conseguir aprender para cursar $4^{\circ}$ y obtener el título. Un 13,32\% responde que, "no están seguros" o "muy poco seguros" y, un 10\%, están "poco seguros" de conseguirlo. Este dato es muy importante, ya que tienen expectativas de éxito futuro y se ha conseguido aumentar la autoestima académica de casi el 80\% del alumnado que cursa PMAR, pero, en otra pregunta, sólo el $40 \%$ afirma que le irá "bien" o "muy bien" en $4^{\circ}$, el otro $60 \%$, no está muy seguro de que les vaya a ir bien el curso; siendo contradictorio con los resultados obtenidos en el anterior ítem. Las orientadoras, en cambio, haciendo alusión al alumnado de un curso anterior, ven cómo, aunque sus notas sean aceptables, ahora en $4^{\circ}$, en general, sólo dos aprueban, con suficiente "raspado", y, otros dos, suspenden y les cuesta mucho trabajo (Orientadora IES "MAC"). Los resultados también bajaron en el IES “TOG”, porque no se pueden establecer grupos de $4^{\circ}$ de Aplicadas en todas las materias por falta de profesorado y de horario en el centro. Han tratado de paliar esta dificultad cambiando los instrumentos de evaluación, utilizando material de apoyo, y otras estrategias, pero, en definitiva, afirma su Orientadora, que el nivel de partida de estos estudiantes está muy por 
debajo, y, en dos años no se puede equiparar, ya que, de ser así, no se estarían produciendo problemas entre el alumnado de $4^{\circ}$ que cursó PMAR.

\section{Conclusiones}

Podemos resumir el procedimiento y el perfil del alumnado del siguiente modo: el Equipo Docente propone al alumnado, se hace una evaluación psicopedagógica previa por parte del Departamento de Orientación, la familia y el alumno debe estar de acuerdo y dar su consentimiento, tanto para la realización del informe, como para la incorporación al programa; el alumnado debe haber repetido al menos una vez y debe tener dificultades en el aprendizaje, que no sean ocasionadas por falta de interés o esfuerzo. No obstante, detectamos un pequeño porcentaje de alumnado que no cumple este último requisito, incluso un estudiante que afirma que no había repetido ningún curso.

Por tanto, se nos plantea la siguiente duda: ¿el alumnado que cursa PMAR accede a éste porque no alcanza el nivel deseado, teniendo problemas de aprendizaje, que no son debidas a una falta de estudio o esfuerzo, o, accede a dicho programa el alumnado que no se esfuerza en aprender?

Esta pregunta nos lleva a desconfiar del proceso de selección de los centros, es decir, si cumplen o no con la legislación vigente, dado que no sabemos si el alumnado que accede al mismo lo hace porque, realmente muestra dificultades de aprendizaje o, como vía de escape para el profesorado de aquellos estudiantes que causan ciertos problemas en clase, en cuyo caso, no se estarían cumpliendo los requisitos de acceso previstos por la ley. Si esto se corroborase con nuevas investigaciones más amplias, tendríamos que replantearnos la necesidad de otras medidas para este tipo de alumnado, que está hastiado del modelo educativo que tenemos, y dejar de utilizar el PMAR, ahora, y el PDC, antes, como "cajón desastre" donde derivar al alumnado que no tiene motivación intrínseca para estudiar.

Haciendo referencia a la legislación vigente sobre el acceso del alumnado a dicho programa, podemos decir, de modo general, que los centros no suelen adecuarse a la ley en su totalidad, como ejemplo de ello tenemos el IES "ALC", "TOG" y "MAC". En estos institutos se selecciona al alumnado con materias pendientes, como incluye la legislación, sin embargo, estos suspensos se deben a una falta de estudio y/o esfuerzo, por lo que tenemos un claro ejemplo de que la mayoría del alumnado no se ajusta al perfil propuesto en la misma.

Por otro lado encontramos discrepancias en la percepción sobre la aceptación de las familias del alumnado a su incorporación al PMAR: los orientadores observan que hay algunas reticencias, padres y madres que prefieren utilizar otras vías de atención a la diversidad, porque consideran que optar por el PMAR, disminuirá las expectativas académicas de sus hijos e hijas, sin embargo, los estudiantes no son conscientes de ello, o no lo perciben así, ya que la mayoría considera que sus familias están contentas de que estén cursando el programa.

Haciendo mención a los elementos del currículum, en el ámbito del PMAR, podemos decir que, tanto los objetivos, como los contenidos que se establecen en el programa, son los mismos que los de la Educación Secundaria Obligatoria, sin embargo, la metodología utilizada por el profesorado cambia totalmente, es decir, en PMAR el profesorado tiene la obligación de atender a la diversidad que se encuentra en las aulas, por lo tanto, hay una atención más personalizada, adaptaciones en las tareas, se respetan los ritmos de aprendizaje del alumnado y el empleo de prácticas y actividades más dinámicas y motivadoras que ayuden a incidir positivamente en el rendimiento y resultados del alumnado.

En cuanto a los criterios de evaluación, la mayoría de los centros confirman que dichos criterios sufren ciertas modificaciones en cuanto a porcentajes e instrumentos. Esto es debido a que muchos de los objetivos no son posibles alcanzarlos cuando estamos hablando de atención a la diversidad y de niveles por debajo de la media. Por lo tanto, los criterios de evaluación son modificados por una evaluación continua, donde se atiende más al esfuerzo y a los logros conseguidos que a las mismas 
calificaciones, aunque éstas, también sean importantes.

No obstante, podemos destacar del PMAR, como algo positivo, que el alumnado, desde el principio, se muestra favorable ante la propuesta de acceder al programa, así como la importancia de que, algunas de las asignaturas, sean impartidas en grupos ordinarios, consiguiendo, de este modo, una normalidad e integración. La mayoría destaca beneficios del agrupamiento.

Para concluir, casi la totalidad del alumnado que ha cursado PMAR, está bastante satisfecho con el ambiente de clase, rendimiento, resultados y expectativas, mostrándose contentos ante la oportunidad que se les brinda con dicho programa. En cuanto a la opinión del profesorado sobre, si los alumnos tienen el nivel necesario para optar a otro tipo de formación educativa, podemos decir que las opiniones han sido muy dispares. La mayoría del profesorado cuestionado considera que el alumnado que ha cursado este tipo de programas tiene menos posibilidades para desarrollar otro tipo de estudios, y creen que lo más aconsejable es el acceso a la Formación Profesional de Grado Medio, con el objetivo de que puedan especializarse, y, entrar en el mundo laboral.

En definitiva, hay luces y sombras respecto a esta medida, al igual que en investigaciones anteriores (Escudero, 2006; Navarro, 2006, Castro y Corujo, 2006), las primeras son puestas de manifiesto por el alumnado, principalmente, y algunos docentes y orientadores; las sombras aparecen cuando se compara el PMAR con el PDC, ya que, no completa la etapa de ESO hasta $4^{\circ}$, no tiene un carácter finalista, sino reparador, aunque con tiempo insuficiente para recuperar las dificultades acumuladas del alumnado. No obstante, es pronto para valorarla de forma cierta, y en su justa medida, y menos aún, si los requisitos de acceso se saltan en algunos casos, como ocurría también con los PDC. Sería necesario profundizar en estos temas y realizar investigaciones más amplias y de seguimiento del alumnado que los cursa para conocer realmente si cumple los objetivos con los que la legislación los propuso.

\section{Referencias}

Adame, M.T., \& Salvá, M. (2010). Abandono escolar prematuro y transición a la vida activa en una economía turística: el caso de Baleares. Revista de Educación, 351, pp. 185-210.

Andreú, J. (2000). Las técnicas de análisis de contenido: una revisión actualizada. Fundación Centro Estudios Andaluces, Universidad de Granada, 10(2), pp. 1-34. Recuperado el 4 de febrero de 2018 de: http://public.centrodeestudiosandaluces.es/pdfs/S200103.pdf.

Aramendi, P., Vega, A., \& Santiago, K. (2011). Los programas de atención a la diversidad en la Enseñanza Secundaria desde la perspectiva de los estudiantes. Revista de Educación, 356, pp. 185209.

Booth, T., \& Ainscow, M. (2000). Index for inclusion. Centre for Studies on Inclusive Education (CSIE). Bristol: UK.

Castro, L., \& Corujo, M.C. (2006). Los Programas de Diversificación Curricular a juicio de los Orientadores/as de los Institutos de Educación Secundaria. Revista Mediodía, 4, pp. 1-15.

Decreto 111/2016, de 14 de junio, por el que se establece la ordenación y el currículo de la Educación Secundaria Obligatoria en la Comunidad Autónoma de Andalucía. (BOJA de 28 de junio de 2016).

Escudero, J.M. (2006). Fracaso escolar y exclusión educativa. Profesorado. Revista de Currículum y Formación de Profesorado, 13(3), pp. 3-9.

Fernández, J.M. (2006). ¿Educación inclusiva en nuestros centros Educativos? Sí, pero ¿Cómo? Contextos Educativos, 8(9), pp. 135-145. doi: 10.18172/con.561.

Herrera, M. (2002). La técnica de encuesta en Investigación Social. En López, F., \& Pozo, T. (Coords.), Investigar en Educación Social (pp. 107-116). Sevilla: Junta de Andalucía. Consejería de Relaciones Institucionales.

Ley Orgánica 1/1990, de 3 de octubre de Ordenación General del Sistema Educativo. (BOE de 4 de octubre de 1990).

Ley Orgánica 2/2006, de 3 de mayo, de Educación. (BOE de 4 de mayo de 2006).

Ley Orgánica 8/2013, de 9 de diciembre, para la mejora de la calidad educativa. (BOE de 10 de diciembre de 2013). 
MECD (2013). Objetivos Educativos Europeos y Españoles. Estrategia Educación Formación 2020. Informe español 2013. Recuperado el 6 de febrero de 2018 de: http://www.mecd.gob.es/dctm/inee/indicadores\%E2\%80\%90educativos/informeet20202013. pdf?documentId $=0901 \mathrm{e} 72 \mathrm{~b} 81732 \mathrm{dc} 8$.

MECD (2016). Datos y Cifras: Curso escolar 2016-2017. Madrid: Secretaría General Técnica, Subdirección General de Documentación y Publicaciones. Recuperado el 5 de febrero de 2018 de: http://www.mecd.gob.es/servicios-al-ciudadano-mecd/dms/mecd/servicios-al-ciudadano$\mathrm{mecd} /$ estadisticas/educacion/indicadores-publicaciones-sintesis/datoscifras/Datosycifras1617esp.pdf.

Mena, L., Fernández, M., \& Rivière, J. (2010). Desenganchados de la educación: procesos, experiencias, motivaciones y estrategias del abandono y del fracaso escolar. Revista de Educación, Extraordinario 2010, pp. 119-145.

Navarro, R. (2006). Los Programas de Diversificación Curricular, ¿qué opinan los alumnos que los han cursado? Revista Española de Pedagogía, 233, pp. 105-122.

Nieto, J.M. (2004). La construcción de la discapacidad de alumnos en riesgo de exclusión vista desde los centros y el profesorado. En Murillo-Estepa, P., López-Yáñez, J., \& Sánchez Moreno, M. (Coords.), Actas Octavo Congreso Interuniversitario de Organización de Instituciones Educativas (pp. 281-290). Sevilla: Universidad de Sevilla, Servicio de publicaciones: Universidad de Sevilla, Departamento de Didáctica y Organización Escolar.

Ortega, I. (2010). Los agrupamientos flexibles intranivel como medida de atención a la diversidad. Revista digital para profesionales de la enseñanza, 11, pp. 1-7.

Parrilla, A., Susinos, T., Gallego-Vega, C., \& Martínez, B. (2017). Revisando críticamente cómo investigamos en educación inclusiva: cuatro proyectos con un enfoque educativo y social. Revista Interuniversitaria de Formación del Profesorado, 89(31.2), pp. 145-156.

Real Decreto 1105/2014, de 26 de diciembre por el que se establece el currículo básico de la Educación Secundaria Obligatoria y del Bachillerato. (BOE de 3 de enero de 2015).

Tarabini, A., \& Montes, A. (2015). La agenda política contra el abandono escolar prematuro en España: la LOMCE contra las evidencias internacionales. Revista de la Asociación de Inspectores de Educación en España, 23, pp. 1-20. Recuperado el 6 de febrero de 2018 de: https://avances.adide.org/index.php/ase/article/view/20/21.

Vallejo, M., \& Bolarín, M. (2009). Los programas de atención a la diversidad y los centros de educación secundaria: claves organizativas, recursos, adscripción del profesorado y valoración de los programas por el profesorado. Profesorado. Revista de Currículum y Formación de Profesorado, 13(3), pp. 143-155. 
